\title{
Protocol
}

Full title: A pilot, randomised, assessor-blinded trial comparing kinesiology tape (lift method) with compression (compress method) for hand oedema post trauma/surgery.

Working title: Is kinesiology tape effective in reducing swelling after a hand injury or surgery?

Trial registration: Awaiting ISRCTN trial identifier.

Protocol version: $30.05 .17 \mathrm{v} 1$

IRAS ID: 228812

Funding: Leanne Miller $(\mathrm{Cl})$ is funded by a National Institute for Health Research and Health Education England Clinical Doctoral Research Fellowship (CDRF-201405-064).

This protocol presents independent research funded by the National Institute for Health Research (NIHR) and Health Education England. The views expressed are those of the authors and not necessarily those of the NHS, the NIHR or the Department of Health.

\section{Principal and Chief Investigator:}

Leanne Miller

NIHR/HEE Clinical Doctoral Research Fellow, MSc, BSc (Hons) Occupational

Therapy

University of East Anglia

Norfolk and Norwich University Hospital NHS Trust

\section{Co-investigators:}

Professor Christina Jerosch-Herold DipCOT, MSc, PhD

Reader in Occupational Therapy

School of Health Sciences

University of East Anglia

Professor Lee Shepstone

Professor of Medical Statistics

Norwich Medical School

University of East Anglia

\section{Contributors:}

Members of the Patient Advisory Committee; Jacqueline Romero (PPIRes Project Manager), Ann Dibben, Pauling Spedding, Jon Craigie and Chris Dell. 


\section{Introduction:}

\section{Background}

Oedema is an abnormally large accumulation of interstitial fluid [1] which collects at the site of injury in the healing phase but which can be slow to dissipate. In healthy tissue there is a balance between the vascular and lymphatic systems. Excessive tissue fluid is rare because arm movements create a force pushing fluid towards the axilla (arm pit).

Following hand trauma or surgery there is increased capillary filtration and reduced lymphatic drainage. Lack of normal limb movement and inactivity results in impaired venous return, and, increases hydrostatic and capillary pressures. Whilst oedema is part of the normal inflammatory response [2] its form alters over time which has implications for how it is treated. In the primary inflammatory phase (3-14 days) oedema is made up of water and dissolvable electrolytes, is soft and easy to mobilise. This type of oedema rarely causes adhesions, but can restrict range of motion. Basic first aid principles (RICE- Rest Ice-Compression-Elevation) are sufficient to reduce this type of swelling. [3,4]

As oedema progresses to a sub-acute phase (2- 6 weeks) the fluid is depleted in nutrients and has increased protein content making it more viscous, resulting in inelasticity and thickening of the tissues. Clinically, this is where problems can start and therefore is the focus of this study. The therapist's objective is to reduce oedema as effectively and quickly as possible in order to prevent long-term functional disability. Prolonged oedema has a negative impact on joint range of motion, soft tissue mobility, quality of scar tissue formation, hand function, strength, and appearance of the hand. Where hand oedema is prolonged, a patient's recovery is delayed. This requires more frequent out-patient appointments, delays in return to work and results in difficulties with daily activities.

The management of oedema is a complex intervention and involves a combination of therapeutic methods prescribed as a package of care as oppose to isolated treatments. These can include wearing a compression glove (intermittently during the day and overnight), elevating the hand above the level of the heart (when able during the day or on pillows overnight), completing exercises whilst the hand is elevated, and massaging the hand over the glove or when the glove has been removed. However, an alternative method for reducing hand oedema exists in the form of an elasticated tape called kinesiology tape. Kinesiology tape is becoming more popular for hand oedema management and is already widely used in NHS clinical practice however there is limited empirical evidence to support its use in the reduction of sub-acute oedema.

\section{Rationale:}

The evidence on effectiveness of Kinesiology tape in the management of sub-acute oedema is limited. Three studies have evaluated the effect of Kinesiology taping in various cohort; following leg lengthening surgery [5], after open reduction internal fixation (ORIF) of mandibular fractures [6] and in patients with hand oedema after an 
acute stroke [7]. The lower limb study [5] found that both

kinesiology tape and lymphatic massage significantly reduced

lower limb oedema in patients post leg lengthening surgery. However the use of kinesiology tape resulted in a significantly faster reduction of the oedema compared to standard lymphatic massage. The authors conclude that due to the paucity of trials evaluating the effectiveness of kinesiology taping in the treatment of oedema of the limbs, further prospective studies are required. The mandibular surgery study [6] found a statistically significant difference in the kinesiology tape group $(p<0.001)$ however no confidence intervals were given. The study which looked at hand oedema [7] showed eight of the $9(88 \%)$ patients in the experimental group had a reduction in oedema with one patient having an increase in oedema. The reductions at both the hand and wrist level were small and there was no statistically significant difference between the 2 groups. In the control group, a median negative change indicated oedema worsened over the 6 day trial, despite receiving therapy which included positioning, active and passive exercises.

All studies have methodological weaknesses including small underpowered sample sizes, lack of blinded assessors, poor retention [7], unaccounted missing data [7], lack of a sham application of tape in the control arm which was possible to blind patients to their treatment allocation and unconventional application of tape for the management of swelling. Lack of detail in the reporting of the studies such as method of randomisation raises further doubts about the quality.

Despite the lack of good quality studies Kinesiology tape is widely used by hand therapists. Clinical anecdotal evidence that it 'seems to work' and patients reporting a high level of satisfaction with this intervention compared to other methods such as the Lycra glove suggest that this method may show potential. There is an urgent need to obtain empirical evidence on the effect of Kinesiology tape and compare this with other established interventions for oedema after hand injury. A definitive (phase III) randomised clinical trial is premature as there is a need to obtain preliminary data on effect sizes, most appropriate primary and secondary outcomes and to test the feasibility of the methods.

\section{Patient and Public Involvement in Research (PPI):}

The basis for this study arouse from a clinical dilemma on how to manage sub-acute hand oedema. This was also shared by patients who reported uncertainty around the efficacy and effectiveness of different oedema management methods prescribed by hand therapists in the department. The Public and Patient Involvement in Research (PPIRes) project which is hosted by South Norfolk Clinical Commissioning Group helped to form a patient advisory group (PAG) for this programme of research. Four current or previous hand therapy patients form this group and during meetings or email exchange have assisted in the protocol development and patient information documents to ensure transparency and understanding. 
Aims:

1. To assess the appropriateness and patient acceptance of using either method for oedema control post injury or surgery.

2. To assess the feasibility of the data collection methods, assessor blinding and recruitment strategy.

3. To assess likely adherence to treatments, and feasibility of using a patient completed adherence diary.

4. To obtain information to inform a sample size calculation for a definitive trial.

5. To obtain an initial estimate of the effect of the intervention relative to control treatments.

Trial design: A pilot parallel group randomised controlled trial.

\section{Methods:}

\section{Study setting:}

This study will be set in the hand therapy department at the Norfolk and Norwich University Teaching Hospital NHS Foundation Trust.

\section{Eligibility criteria:}

\section{Inclusion Criteria:}

Patients referred to out-patient hand therapy department at the Norfolk and Norwich University Hospital who are aged 18 years and over, able to give informed consent and for whom treatment of sub-acute* hand oedema is indicated, as confirmed by their treating therapist.

*Sub-acute refers to oedema which is present between days 3 post injury or surgery up to 6 weeks.

\section{Exclusion Criteria:}

1. Patients more than 6-weeks after their injury and/or surgery whose oedema is not classified as sub-acute.

2. Patients who are within the specified sub-acute timeframe but have already commenced oedema management treatments.

3. Patients with diagnosed lymphedema, acute infections, deep vein thrombosis, blood clot or haematoma, active cancer, chronic heart failure, cardiac problems or renal dysfunction/failure/kidney disease, pulmonary problems or any other factor (physical or mental health) that may affect the patient's ability to adequately and safely monitor the use of tapes or gloves.

4. Patients in the first 4 weeks of tendon repairs where removal of their splints in order to apply a glove would be contraindicated. 
5. Patients who do not have someone available to assist in the reapplication of kinesiology tape every 5-5 days, and who do not feel confident to reapply the tape themselves.

6. Fragile skin (elderly and long term steroid use) and open wounds.

7. Patients with excessive amount of hair on their hand/forearms who would find the tape too uncomfortable to be removed every 3-5 days and who may find the pull on the hairs from the tape an unacceptable side effect of using this method. (Often patients will shave the section where the tape is being applied if this is deemed necessary and acceptable to the patient)

\section{Interventions:}

The intervention and control arms of this pilot randomized controlled trial were agreed upon during a three-round internet mediated Delphi Consensus Method with 8 hand therapy experts [unpublished].

Control arm (Treatment As Usual): Participants in this group will receive Treatment As Usual (TAU) which is some form of compression (called a compression glove, compression finger sleeve, or elasticated finger wrap, brand name Coban $\AA$, for the purposes of the patient information leaflets) plus elevation and massage.

Intervention arm (Trial Treatment): Participants in this group will receive the Trial Treatment (TT) which is Kinesiology tape (called 'Elasticated Tape' for the purposes of the patient information leaflets) plus elevation and massage.

\section{Control: (refer to patient instruction booklet)}

Compression for hand oedema is usually achieved through Lycra gloves which exerts around $35+/-5 \mathrm{mmHg}$ pressure on the tissues of the hand [3]. The garment acts as an external counter pressure [3] which compensates for the inelasticity of oedematous tissues, and, therefore improves circulatory efficiency by facilitating venous and lymphatic flow [2]

Compression in the form of an; oedema glove, Lycra digital sleeve or Coban® wrap (See Figure 1) will be issued to patients with advice regarding wearing. They are able to remove these for hygiene purposes but must wear the compressive garment during any prescribed exercises and function for 20-24 hours a day. Patients must be advised to check for tip vascularity to ensure the compression is not/does not become too tight, and, remove if this is the case. Patients will also be advised in methods of elevation and massage as detailed above.

Massage techniques are used to stimulate the lymphatic system [2]. Different methods are documented in the literature which employ various degrees of force or pressure on the skin directing the oedema towards regional lymph nodes. Traditional 'retrograde massage' uses a moderate force 'milking' action but is considered too 
aggressive for the delicate lymphatic system to cope with and has recently been questioned [4]. Instead, a light tractioning of the skin is proposed in a longitudinal direction to produce a stretch reflex to the skin [8]. The type of massage agreed during the Delphi Consensus Method for sub-acute oedema post trauma or surgery was retrograde (distal to proximal direction) massage (85.7\% consensus) which was defined as being firm pressure on the skin and underlying tissues. Experts agreed this should be done for 5-10 minutes, 3-6 times a day (less if unable to tolerate or if a smaller area was affected) for at least 2 weeks or until the swelling had resolved.

Elevation permits gravity to assist with the drainage of oedema from the distal limb [4]. Elevation alone [9] is not effective in reducing oedema, but, is recommended in combination with other modalities. Experts agreed that the hand should either be elevated actively, without being held in place, during the day, or with a Bradford sling or propping the arm on pillows for day or night time elevation. Elevation should be advised "as much as possible" during the day and night when the hand is not being used for function, hygiene and exercises. This should be continued until the patient and therapist mutually agree the oedema has subsided or unless discontinued due to contraindications.

\section{Intervention: (refer to patient instruction booklet)}

Kinesiology tape (refer to figure 1) is designed to mimic the elastic properties of the skin by lifting the skin to allow greater interstitial space and encourage lymphatic drainage. In contrast to the traditional compression method it is designed to push the fluid proximally into the venous and lymphatic system [10]. The tape is said to be unique in that it mimics the elastic properties of the skin and it's wave-like grain provides a pulling force to the skin creating more space by lifting the fascia and soft tissues under the areas where it is applied [11] This multi-functional tape can be applied anywhere on the face or body. The benefit of using it in the hand, unlike an oedema glove or other form of compression, is that it leaves the majority of the skin surface free for sensory feedback which is essential for functional use. It can also be worn in water. As the tape is elastic and stretches up to $55-60 \%$ of its length it also allows for unrestricted movement [11,12]. Experts agreed that the tape should be applied in a fan shape with proximal anchor or volar and dorsal strips with finger holes. There should be no tension at the anchor and $0-25 \%$ tension of the central tape. The tape should be worn full time for 3-5 days until needing to be replaced or sooner if the tape is dirty. A 23-hour rest period can be utilised between applications but is not essential if there has been no issues. The tape needs to be removed in case of skin irritation.

Massage and Elevation: as detailed above for control TAU. 
Kinesiology tape, the oedema glove, Lycra digital sleeve and

Coban $\AA^{\circledR}$ hold CE markings and are being used for their intended purpose, Medicines and Health products Regulatory Agency (MHRA) devices approval is therefore not required.

Treatment will be discontinued or modified at the discretion of the treating hand therapist in the following cases;

- Worsening oedema or other relevant symptoms (pain, stiffness) as assessed by the treating hand therapist via visual assessment, goniometry range of joint motion or subjective symptom severity reporting.

- If a patient, after starting a treatment, reports they no longer find it acceptable and wishes to discontinue. Details of barriers (such as appearance, cleanliness etc) to using treatments will be captured in the adherence diary and patient acceptability questionnaire.

Protocol deviations will be recorded by the hand therapists on a study form detailing what modification has been made to the treatment protocol and when.

Strategies to improve adherence to intervention protocols include monitoring by a hand therapist during arranged out-patient hand therapy appointments on an individual clinical need basis. Adherence to prescribed treatments will be monitored with the use of an adherence diary.

Permitted concomitant care or interventions includes: anti-inflammatory medication to be taken as prescribed, cryotherapy or heat treatments, injury specific hand therapy exercises and advice on managing an injury/ post-surgical care.

\section{Efficacy Outcome:}

The primary efficacy outcome will be the change in hand volume from baseline to follow up assessment. This will be objectively assessed using the volumeter (water displacement method) and subjectively via the Oedema Rating Scale (ORS). These were shown to be the most responsive outcome measures from an observational study of 73 patients with hand oedema based on data from baseline to 4 week assessment.

Secondary outcomes are: (i) function assessed using the Patient Evaluation Measure [4] and (ii) quality of life assessed with the EQ-5D-5L [5].

The number of visits to hand therapy, grade of hand treating hand therapist and total time treating oedema will be also be recorded to obtain preliminary data on healthcare use and cost.

\section{Participant timeline:}


Patients will be assessed at baseline prior to randomisation and again at 4 weeks and 12 weeks post randomisation. Refer to patient flow diagram.

\section{Sample size:}

As a pilot study, principally conducted to assess the suitability of the chosen research methods, the sample size is not based upon the principals of statistical precision or statistical Power for hypothesis testing. Instead, we aim to recruit 100 subjects in a 6 month period which we believe to be practical (see below recruitment section). Based upon the attrition rate of a recently conducted observational study (IRAS 209952), we anticipate a loss to follow-up of between $20 \%$ and $30 \%$, thus providing 70 to 80 completing participants. This is in excess of the rapidly establishing minimum figure of around 60 subjects for a pilot or feasibility study.

\section{Recruitment:}

Patients will be recruited from the out-patient hand therapy department at the Norfolk and Norwich University Hospital upon identification of oedema where there is a clinical need to treat this. The hand therapy department receives up to 500 new referrals every month. It is estimated that at last $80 \%$ of these patients will require some form of oedema management intervention. A recent observational study (IRAS 209952) successfully recruited 100 hand therapy patients with hand swelling between January and April 2017.

Strategies for achieving adequate participant enrolment to reach target sample size include; allowing a sufficient recruitment period of up to 6 months, educating the hand therapy team and ensuring their commitment to recruiting eligible participants and utilising feedback from my patient advisory group to help develop accessible patient documents, ensuring transparency of participants' involvement to reduce loss to follow up.

\section{Methods: Assignment of interventions}

\section{Allocation}

Sequence Generation: Participants will be randomly allocated to either the intervention of control arm. Allocation will be on a 1:1 basis (i.e. equal numbers in each arm). The allocation sequence will be blocked randomised (with random block lengths of 2,4 or 6) generated by the trial statistician (LS). No stratification will be used

Allocation concealment mechanism: sequentially numbered opaque sealed envelopes will be employed. These will be kept in a locked box inside a lockable storeroom in the hand therapy department. The allocation sequence and preparation of the numbered envelopes will be done by the trial statistician (LS) at the University 
of East Anglia. Details of this process will not be made available to the Hand Therapists who are assigning the interventions to participants or to the CI (LM) who will enrol participants.

Implementation The allocation sequence will be generated by a medical statistician (LS). Patients will be identified by the hand therapists as being suitable for the study. If the patient agrees to take part they will then be enrolled onto the study by the $\mathrm{CI}$ (LM) who will confirm eligibility and complete the consent process. Baseline measurements will then be taken by the $\mathrm{Cl}$ (LM). The treating hand therapist will then assign the participant to the trial intervention arm according to the next numbered envelope. This allocation will be kept hidden from the CI (LM).

\section{Blinding:}

It is not possible to blind the patient in this trial. However the assessor (LM) will be blinded as a means of minimising bias. Patients will be asked by their treating therapist not to reveal their treatment allocation during any follow up assessment and will be instructed to remove all oedema management garments prior to being seen by the assessor. However, unintentional unblinding may be possible due to marks left on the skin by the glove or kinesiology tape. The $\mathrm{Cl}$ will record when she believes she has been unblinded and how. Where blinding has been maintained the $\mathrm{Cl}$ will be asked to guess the group allocation and compare against chance. We will establish whether it is feasible for the assessor to remain truly blinded or whether other methods to maintain assessor blinding need to be explored.

\section{Methods: Data collection, management and analysis.}

One blinded assessor (LM) will collect all outcomes at baseline, 4 and 12 weeks.

Volumetry, which uses Archemedes principle of water displacement is referred to as the "gold standard" method of measuring hand size. It has very good intra-rater $(\mathrm{ICC}=0.99)$ and intra-rater (ICC $=0.99)$ reliability [15] and responsiveness [16]. The manufacturer's guidelines will be followed [17] for setting up the Volumeter and upper limb positioning. The water temperature will also be monitored and maintained between 18-24 degrees [18]. One assessment of the oedematous hand is taken, taking around 2 minutes, with the amount of displaced water being recorded in $\mathrm{ml}$.

The Oedema Rating Scale (ORS) was designed by members of the patient advisory group, this consisted of 4 current or previous hand therapy patients. The relative responsiveness of this 7-point oedema numerical rating scale which also includes descriptors under each number, was recently compared to an existing validated patient reported outcome measure commonly used with hand therapy patients and was found to be more responsive in a cohort of 73 patients being treated for hand oedema between baseline and 4 weeks. Patients are asked to tick the box which best describes the amount of swelling in their hand or affected area with zero being no swelling and 6 being extreme swelling. This takes around 1 minute to complete. 
The Patient Evaluation Measure (PEM) is a validated patient rated outcome measure [13] which will be used to measure function in this study. This consists of 11 questions on a 0-7 numerical rating scale, expressed as a percentage, which asks patient to rate aspects such as pain, grip, work and activities of daily living, the higher the score, the greater the perceived disability

The EQ-5D-5L, is a development of the original EQ-5D. It is a standardized measure of health status which aims 'to provide a simple generic measure of health for clinical and economic appraisal' [19]. It was designed to improve the instruments sensitivity and reduce ceiling effects however it does not yet have population normative data. It is recommended by the Chartered Society of Physiotherapists to be used to measure change in musculoskeletal outpatient settings [20]. It takes around 2 minutes to complete

The ORS, PEM and EQ-5D-5L are all paper based questionnaires. Results will be recorded by the blinded assessor on a data collection form.

A simple paper diary designed on best practice recommendations [21] will be used to capture data on patients' adherence to their allocated treatment. Whilst the use of diaries pose their own issues in terms of adherence to completing the diary and whether the diary itself increases a patient's awareness of adhering to a treatment, it is crucial to establish if these interventions are acceptable and being used in real life. Apart from stating the allocated treatment group, the diary will be anonymous and patients will be asked to leave them in a box at reception or return them to the researcher in a pre-paid envelope.

Acceptability of an intervention is an important factor in treatment adherence. A brief patient acceptability questionnaire will be completed with all participants at their final follow up assessment (week12) to ask about their experiences of using the prescribed treatment. This questionnaire consists of a set of ten structured questions where the patient is asked to grade aspects such as appearance, ability to complete everyday activities and cleanliness on a 0-10 scale. The final question will be open to allow feedback from the patient on any aspect not covered in the questionnaire. Responses will be documented. This questionnaire can be done over the phone if preferred by the patient. Refer to patient acceptability questionnaire.

Efforts will be made by the $\mathrm{Cl}(\mathrm{LM})$ and the hand therapy team to promote participant retention and complete follow up. These include combining study follow up assessments with arranged hand therapy appointments to reduce travel and cost attending the hospital but also offering to reimburse any participants for additional travel and parking costs if they are unable to combine study assessments with hand therapy appointments. Therapists will record any deviations from the intervention protocols on a health economy data collection form and inform the $\mathrm{Cl}$ of any 
participant who which to discontinue with their study intervention.

These participants will be asked to remain in the study regardless

of changes to their allocated intervention or stopping the treatment in order to complete follow up and analyse according to intention to treat.

Anonymised data will be entered onto an excel spreadsheet using patient ID numbers $(01,02,03$ etc) cells will be programmed to check ranged of data value entered. This spreadsheet will be stored on a password protected UEA laptop. All data will be entered by the $\mathrm{Cl}$ (LM) who has access to the password protected laptop which is for the sole purpose of this programme of research.

\section{Statistical methods}

A general linear model will be used to estimate the effect of Kinesiology tape relative control, with respect to the efficacy outcomes. This will include the baseline value as a co-variate and treatment arm as a fixed effect. The between group estimate will be estimated with 95\% confidence intervals (though as a pilot study it is unlikely that any conclusion regarding efficacy would, or indeed, should, be reached). This will be based upon the intention-to-treat population though there are no plans for imputation of missing data. There are currently no plans for any subgroup analyses.

The standard deviation will be calculated for each efficacy outcome, together with a $95 \%$ confidence interval, assuming a Normal distribution. The level of missing data for each of the outcomes will be assessed and compared with baseline characteristics (i.e. to identify which groups of subjects, if any, are less likely to return full data).

Descriptive analyses will be used to assess 'patient flow', particularly estimating the proportion of eligible patients consenting to take part, the frequency of precluding eligibility criteria, and the frequency of loses to follow-up, including active withdrawals (with reason where available). Each proportion will be calculated with a $95 \%$ confidence interval.

Patient reported adherence will be calculated for each participant as a proportion of the 'total compliance' which would be the frequency and duration advised by the hand therapist and documented in the patient instruction manual. This will be summarised as a mean with $95 \%$ confidence interval, assuming a Normal distribution.

\section{Methods: Monitoring}

Due to the short follow-up period of this study and the minimal risks associated with the interventions a data monitoring committee will not be needed. The CI (LM) will be in regular contact with her supervisors and patient advisory group to discuss any 
issues, if required, however it is unlikely that the trial protocol will need modifying during the period of the trial as a results of interim data.

All unexpected adverse reactions or events will be formally reported in accordance with Standard Operating Procedure (SOP 205 v2.3) at the Norfolk and Norwich University Hospital using the Serious Adverse Events (SAE) report form v1.3. All documents are available on the hospital research and innovation website.

\section{Ethics and dissemination:}

Approval will be obtained from the Research Ethics Committee (REC), Health Research Authority (HRA) and the Research and Development Department at the Norfolk and Norwich University Hospital prior to commencement of the study. In the event of significant modifications to the protocol the above agencies will be consulted in order to gain formal approval.

Initial informal assent will be gained from a hand therapist. The CI (LM) will then obtain informed written consent from participants after they have read the Patient Information Sheet (PIS) and had the opportunity to ask any questions. The participant will be given a copy of their signed consent form. The $\mathrm{Cl}$ (LM) is GCP trained and has experience of gaining informed written consent.

Any required personal/identifiable information about enrolled participants needed for the duration of the study (name, hospital number) will be written onto hard copies of study forms (case report form and data collection forms) and will be stored securely in locked filing cabinets or in cupboards in the Hand Therapy department of the Norfolk and Norwich hospital and/or UEA office. Only members of the direct medical or therapy team involved with a patients care will know about their involvement in the study. Identifiable patient details will not be discussed with members of the supervisory or patient advisory group. Data will be anonymised for electronic data entry and analysis. Electronic files on university computer/laptop will be password protected and backed up daily via the UEA IT back-up systems.

The NHS Code of Confidentiality and Data Protection Act will be followed.

The final anonymised trial dataset will be accessed by the $\mathrm{Cl}$ and her supervisor team if required to assist with data analysis.

Primarily the results of this observational study will form part of my doctoral thesis however it will also inform updates in local departmental oedema guidelines, will be presented, at national and international hand therapy conferences and published in a relevant open-access peer-reviewed journal. Those patients who confirmed they would like to receive a report of the finding will be posted a basic science summary which will undergo patient and public involvement review. 
Figure 1: Methods of compression (control arm), elevation (standard treatment) and kinesiology tape application (intervention arm)

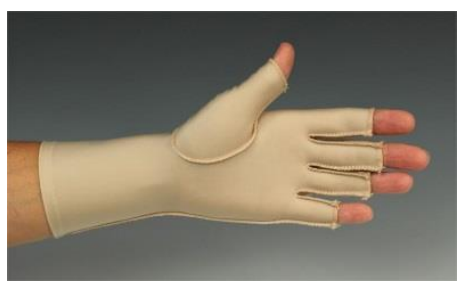

Oedema glove- open fingertip

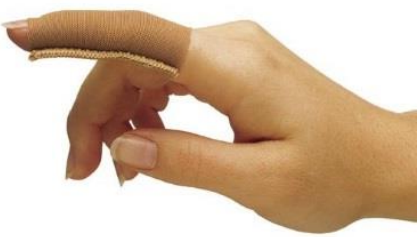

Lycra digital sleeve

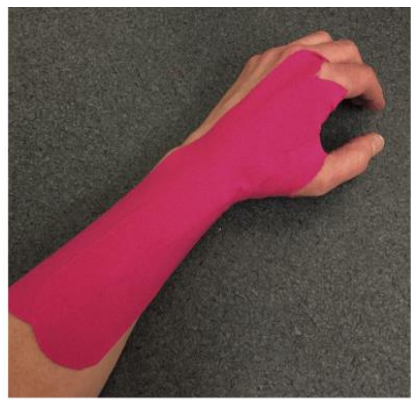

Kinesiology tape- volar and dorsal strip with finger holes

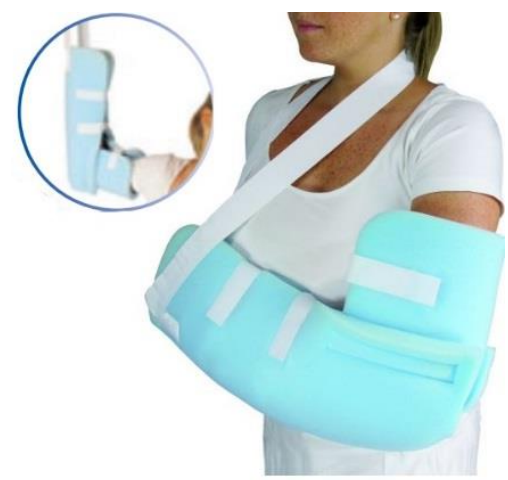

Bradford sling- day and/or night hand elevation

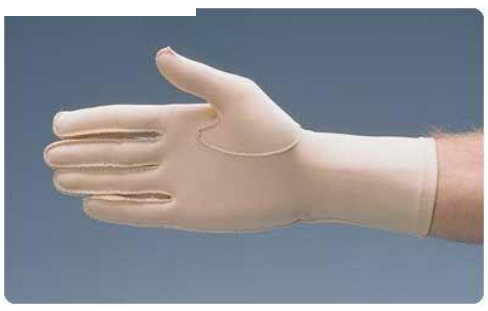

Oedema glove- closed fingertip

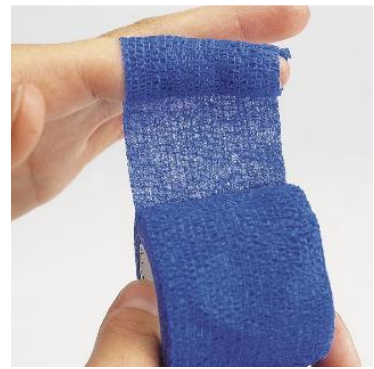

Coban $\AA$ self-adherent wrap

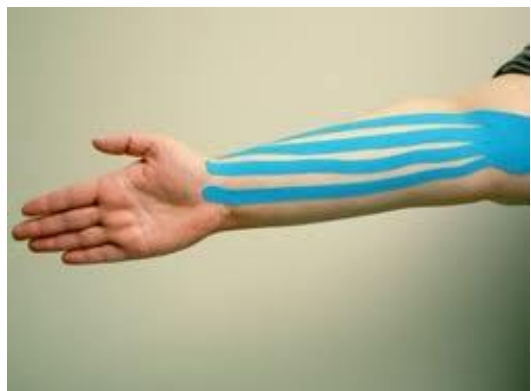

Kinesiology tape- fan with proximal anchor

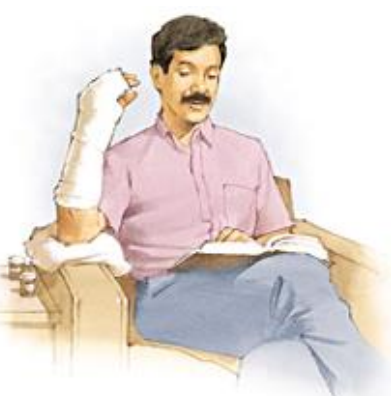

Active elevation $+/$ - pillows for day/night. 


\section{References}

1. Schmidt RF, Thews G eds 1989. Human Physiology 2nd edn. SpringerVerlag. Berlin

2. Villeco J. Edema: A silent but Important Factor. Journal of Hand therapy. 2012:25:153-62

3. Newman G. Which patients with arm oedema are helped by intermittent external pneumatic compression therapy. Journal of Royal Society of Medicine. 1988:81:377-9

4. Pedretti LW, Zoltan PJ.Occupational Therapy Practice skills for Physical Dysfunction. 4th ed 1996. Mosby, California.

5. Bialoszewski D, Wozniak W and Zarek S. Clinical efficacy of Kinesiology Taping in Reducing Edema of the Lower Limbs in Patients Treated with the llizarov Method-Preliminary Report. Orthopedia Traumatologia Rehabiliacja. $20091(6) ; 1146-54$.

6. Ristow O, Hohlwegg-Majert B, Kehl V et al. Does Elastic Therapeutic Tape Reduce Postoperative Swelling, Pain and Trismus After Open Reduction and Internal Fixation of Mandibular Fractures. Journal of Oral Maxillofacial Surgery 2013 71:1387-1396

7. Bell A, Muller M. Effects of Kinesio Tape to Reduce Hand Edema in Acute Stroke. Top Stroke Rehabil. 2013; 20(3); 2830-288

8. Zuther EZ. Lymphedema Management. 2nd Edition. New York. NY. Thieme, 2009.

9. Watson-Jones, Sir R. (1955): Fractures and Joint Injuries. Fourth edition. Edinburgh and London: E. \& S. Livingstone Ltd.

10. Kase K, Wallis J, Kase T. Clinical Therapeutic Application of the Kinesio Taping Method. Tokyo: Ken Ikai Co. Ltd 2003

11. Williams S, Whatman C, Hume P, Sheern K. Kinesio Taping in Treatment and Prevention of Sports Injury: A meta-analysis of the evidence for its effectiveness. Sports Med 2012; 42(2) 153-164. 
12. Thelen MD, Dauber JA, Stoneman PD. The Clinical Efficacy of Kinesio Taping for Shoulder Pain: A randomized, Double- Blinded, Clinical Trial. J Orthop Sports Phys Therapy. 2008; 38(7): 389-395

13. Dias JJ, Bhowal B, Wildin CJ, Thompson JR. Assessing the outcome of disorders of the hand. Is the patient evaluation measure reliable, valid, responsive and without bias? J Bone Joint Surg Br. 2001 Mar;83(2):235-40.

14. Herdman M, Gudex C, Lloyd A, Janssen MF, Kind P, Parkin D, Bonsel G, Badia $X$. Development and preliminary testing of the new five-level version of EQ-5D (EQ 5D- 5L). Quality of Life Research.

15. Farrell $\mathrm{K}$, Johnson $\mathrm{A}$, Duncan $\mathrm{H}$ et al. The intertester and intratester reliability of hand volumetrics. Journal of Hand Therapy. 2003; 16:292-9

16. Leard J, Crane B, Mayette $\mathrm{D}$ et al. Responsiveness of the figure of eight tape measurement to detect hand size changes of patients with acute and chronic hand pathologies. The British Journal of Hand Therapy. 2004 13:3 84-90.

17. Creelman G, Creelman J. Hand Volumeter: Directions for Use. Idyllwild, CA: Volumeters Unlimited, 1989.

18. King $T$. The effect of water temperature on hand volume during volumetric measurement using the water displacement method. J Hand Ther. 1993;6:202-4

19. Devlin, N. EuroQuol Group. Office of Health Economics Research 2010.

20. Sephton R, Hough E, Roberts SA, Oldham J. Evaluation of a primary care musculoskeletal clinical assessment service: a preliminary study.

Physiotherapy 2010 12;96(4):296-302.

21. Frost $R$, McClurg D, Brady $M$ et al. Optimising the validity and completion of adherence diaries: a multiple case study and randomised crossover trial. Trials. 2016 17: 489. 